\title{
BMJ Open Social determinants of health associated with the development of sepsis in adults: a scoping review protocol
}

\author{
Christina Machon, ${ }^{1}$ Fatima Sheikh, ${ }^{2}$ Alison Fox-Robichaud (i) ${ }^{3}$
}

To cite: Machon C, Sheikh F, Fox-Robichaud A. Social determinants of health associated with the development of sepsis in adults: a scoping review protocol. BMJ Open 2020;10:e039146. doi:10.1136/ bmjopen-2020-039146

- Prepublication history and additional material for this paper are available online. To view these files, please visit the journal online (http://dx.doi. org/10.1136/bmjopen-2020039146).

Received 06 April 2020 Revised 04 October 2020 Accepted 12 0ctober 2020
A) Check for updates

(C) Author(s) (or their employer(s)) 2020. Re-use permitted under CC BY-NC. No commercial re-use. See rights and permissions. Published by BMJ.

${ }^{1}$ Health Sciences Programme, McMaster University, Hamilton, Ontario, Canada

${ }^{2}$ Life Sciences Programme, McMaster University, Hamilton, Ontario, Canada

${ }^{3}$ Medicine, McMaster University, Hamilton, Ontario, Canada

Correspondence to Dr Alison Fox-Robichaud; afoxrob@mcmaster.ca

\section{ABSTRACT}

Introduction Sepsis, the life-threatening immune response to infection, affects millions of people annually. Understanding of the factors associated with the development of sepsis is crucial for improving population health and public health efforts; in particular, literature exploring the relationship between sepsis and social determinants of health is lacking. This review seeks to establish and amalgamate existing evidence of the relationships between sepsis and the following social determinants: frailty, registration with a family physician, mental illness, alcohol abuse, social support levels, smoking status, illicit drug use disorders, socioeconomic status, gender and race/ethnicity.

Methods and analysis This study will analyse qualitative and quantitative studies using standard processes. The selected social determinants of health and their potential link to adult sepsis will be analysed separately under distinct headings. Findings will be consolidated in a final discussion. PubMed and Medline will be searched for articles published between 1970 and 2020 using search strings combining 'sepsis' and other variations, such as 'septicaemia' with each social determinant of interest. 'Sepsis' and at least one social determinant of interest must be present in a study's title for inclusion in the review; the results of the initial search will be filtered based on predetermined inclusion and exclusion criteria. Evidence from this scoping review will provide information on the impact of social determinants of health on the risk of developing adult sepsis, which can inform clinicians of the various risk factors to consider when admitting patients.

Ethics and dissemination Approval from a research ethics board is not needed for this amalgamation of information from studies for which the primary investigators have obtained their own, respective ethics board approval. Once completed, the review will be submitted for publication in a peer-reviewed journal, and findings will be presented in local and national forums.

\section{INTRODUCTION}

\section{Rationale}

Sepsis, as defined by Singer $e t a l$, is the lifethreatening organ dysfunction caused by a dysregulated host response to infection. ${ }^{1}$ Although sepsis can be difficult to diagnose and thus document, it was estimated that, in 2017, there were approximately 48.9 million

\section{Strengths and limitations of this study}

This scoping review will be the first to focus on any associations that exist between social determinants of health and the development of sepsis in adults.

- This study will investigate both qualitative and quantitative studies, analysing findings from primary research articles written in English and published between January 1970 and December 2019.

- The quality of the literature will be assessed using the Quality in Prognosis Studies and the Grading of Recommendations, Assessment, Development and Evaluation approach, where appropriate.

- The protocol includes a data abstraction table to standardise the data collection process.

- Findings from unpublished and non-commercial published literature and articles written in other languages may be missed.

cases of sepsis worldwide and 11 million sepsis-related deaths, accounting for $19.7 \%$ of all global deaths. ${ }^{2}$ Unsurprisingly, the financial burden of sepsis on healthcare systems is enormous, with an annual estimated cost of US\$24 billion in the USA. ${ }^{2}$ An improved understanding of the factors contributing to the development and progression of sepsis is crucial to improving population health.

WHO broadly defines social determinants of health as "the conditions in which people are born, grow, live, work and age'. An analysis of the degree to which social and economic conditions may hinder the health of certain groups is essential to understanding and improving whole-population well-being. Among their list of internationally pertinent social determinants of health, WHO includes employment conditions, social exclusion and marginalisation, early childhood development and urbanisation. ${ }^{3}$

These social determinants highlighted by WHO informed this scoping review as well as the other components of this research programme (including a retrospective chart review on sepsis hospital admissions). A preliminary search of scoping reviews and 
systematic reviews was conducted of the International Prospective Register of Systematic Reviews, the Joanna Briggs Institute Database of Systematic Reviews and Implementation Reports, The Cochrane Database of Systematic Reviews and the Campbell Library. To date, there has not been a scoping or systematic review published that explores the relationship between social determinants of health and the development of sepsis in adults. This understanding of the social determinants that impact the development of sepsis can inform patient care by alerting healthcare providers to an increased risk of sepsis.

\section{Purpose and objectives}

The proposed scoping review aims to provide knowledge of the relationship between social determinants of health and adult sepsis, in addition to promoting understanding of the various risk factors that clinicians should consider on hospital admission.

\section{RESEARCH QUESTIONS \\ Primary}

- Are there any social determinants of health that are associated with the development of sepsis in adults?

- If so, what are these determinants?

\section{Secondary}

- How might the knowledge of a link between certain social determinants of health and the development of adult sepsis inform current healthcare practices?

The clinical outcome being assessed is a diagnosis of sepsis.

\section{METHODS}

The search will be conducted using PubMed and Medline; the full search string and search terms can be found in online supplemental appendix 1 . Articles will be independently screened by two reviewers-first, for initial screening, and again for eligibility. Afterwards, agreement between reviewers will be assessed to determine which articles are to be included. Any disagreements will be resolved by a third reviewer. Articles will be critically appraised using the Quality in Prognosis Studies (QUIPS) tool $^{4}$ and the Grading of Recommendations, Assessment, Development and Evaluation (GRADE) approach ${ }^{5}$ to assess the quality of evidence within systematic reviews and meta-analyses, where applicable. Unpublished and non-commercial published literature will not be searched.

Data will be abstracted using a charting form (online supplemental appendix 2), indicating the author(s), year, concept, context and results of each outcome measure (sepsis diagnosis).

\section{Inclusion criteria}

This review will include studies of qualitative, quantitative and mixed-methods design. Each study must include 'sepsis' (or synonyms such as 'septicaemia' or 'bacteraemia') and one of the defined social determinants of health including: socioeconomic status, race, illicit drug use disorders, social support levels (independent, living with family or living in a long-term care facility), registration with a family doctor, mental illness, alcohol use, smoking status, frailty and gender (as distinct from sex, which is biological), (see online supplemental appendix 1 for full-search strategy and search terms). The references of eligible studies will be searched for additional literature. Meta-analyses and systematic reviews will be excluded but the references will also be searched for additional studies. All articles must be written in English, involve human participants and be published between 1970 and 2020. These years were selected to attempt to capture most articles that have been published on the subject, while also recognising that recency contributes to article quality. The most recent search date was 29 January 2020.

\section{Exclusion criteria}

Articles will be excluded if they are editorials, commentaries, letters or narrative reviews (narrative reviews will be searched for additional primary studies). Excluded articles also include those that use animal models, participants under the age of 18 years, are written in a language other than English, or are published prior to 1970. Additionally, articles deemed to be of low quality, according to the QUIPS or GRADE criteria, may be excluded with the agreement of all three reviewers.

\section{Patient and public involvement}

Patients and the public were not involved in the planning of this scoping review.

\section{Ethics and Dissemination}

Approval from a research ethics board will not be required for this review. Once completed, the review will be submitted for publication in a peer-reviewed journal, and findings will be presented in local and national forums.

\section{DATA ANALYSIS}

Data will be abstracted as per the charting form in online supplemental appendix 2. As recommended by Levac, Colquhoun and O'Brien, the data analysis stage will be done in three steps: analysing the data, reporting results and applying meaning to the results. ${ }^{6}$ Final summarisation and collation of qualitative findings will be presented thematically (such as the links between socioeconomic status, substance use disorders and delayed seeking treatment for infections or the relationship between low socioeconomic status, health literacy and intensive care unit admission for sepsis), according to each individual social determinant of health (online supplemental appendix 1). Additionally, we will follow Arksey \& O'Malley's approach to providing descriptive numerical summaries for quantitative findings (eg, relative income and risk for intensive care admission with sepsis), presented in tables. ${ }^{7}$ These summaries will include study characteristics such as the 
years of publication, countries of origin and characteristics of the study populations as well as descriptive statistics where available.

\section{DISCUSSION}

This scoping review will be the first review to focus on social determinants of health in the context of sepsis. The vast majority of current research focuses on biological risk factors of developing sepsis. Such risk factors include being 65 years or older, having a weakened immune system, having chronic conditions (eg, cancer, diabetes, lung disease), and being a child younger than 1 year. $^{89}$ Other studies have focused on microbiological risk factors for developing sepsis. For instance, Grion et al found that high density lipoproteins may have a protective effect against sepsis. ${ }^{10}$ While knowledge of biological risk factors is crucial to understanding sepsis development, it does not tell the whole story. Social factors have been found to be associated with the incidence of multiple health conditions, such as cardiovascular disease ${ }^{11}$ and stroke. ${ }^{12}$ Furthermore, they have been found to impact breast cancer stage at diagnosis and subsequent survival. ${ }^{13}$ Given that social determinants of health have been implicated in the progression and development of many health conditions, it stands to reason that they may be involved in the development of sepsis. The discovery of which social determinants affect the development of sepsis is crucial to advancing knowledge to further public health efforts.

\section{Twitter Alison Fox-Robichaud @drfoxrob}

Contributors This protocol, including the appendices, was written by CM (BHSc candidate). FS (BSc candidate) contributed by editing the protocol. AF-R provided general supervision, in addition to assisting with technical editing, language editing and proofreading. All authors approved the final manuscript.

Funding The authors have not declared a specific grant for this research from any funding agency in the public, commercial or not-for-profit sectors.

Competing interests None declared.

Patient consent for publication Not required.

Provenance and peer review Not commissioned; externally peer reviewed.

Supplemental material This content has been supplied by the author(s). It has not been vetted by BMJ Publishing Group Limited (BMJ) and may not have been peer-reviewed. Any opinions or recommendations discussed are solely those of the author(s) and are not endorsed by BMJ. BMJ disclaims all liability and responsibility arising from any reliance placed on the content. Where the content includes any translated material, BMJ does not warrant the accuracy and reliability of the translations (including but not limited to local regulations, clinical guidelines, terminology, drug names and drug dosages), and is not responsible for any error and/or omissions arising from translation and adaptation or otherwise.

Open access This is an open access article distributed in accordance with the Creative Commons Attribution Non Commercial (CC BY-NC 4.0) license, which permits others to distribute, remix, adapt, build upon this work non-commercially, and license their derivative works on different terms, provided the original work is properly cited, appropriate credit is given, any changes made indicated, and the use is non-commercial. See: http://creativecommons.org/licenses/by-nc/4.0/.

ORCID iD

Alison Fox-Robichaud http://orcid.org/0000-0001-9912-3606

\section{REFERENCES}

1 Singer M, Deutschman CS, Seymour CW, et al. The third International consensus definitions for sepsis and septic shock (Sepsis-3). JAMA 2016;315:801-10.

2 Rudd KE, Johnson SC, Agesa KM, et al. Global, regional, and national sepsis incidence and mortality, 1990-2017: analysis for the global burden of disease study. Lancet 2020;395:200-11.

3 World Health Organization. About social determinants of health. Available: http://www.who.int/social_determinants/sdh_definition/en

4 Hayden JA, van der Windt DA, Cartwright JL, et al. Assessing bias in studies of prognostic factors. Ann Intern Med 2013;158:280-6.

5 Guyatt G, Oxman AD, Akl EA, et al. Grade guidelines: 1. IntroductionGRADE evidence profiles and summary of findings tables. $J$ Clin Epidemiol 2011;64:383-94.

6 Levac D, Colquhoun H, O'Brien KK. Scoping studies: advancing the methodology. Implement Sci 2010;5:69.

7 Arksey H, O'Malley L. Scoping studies: towards a methodological framework. Int J Soc Res Methodol 2005;8:19-32.

8 CDC. Sepsis is a medical emergency. time matters, 2019. Available: https://www.cdc.gov/sepsis/what-is-sepsis.html [Accessed 11 Mar 2020].

9 Henriksen DP, Pottegård A, Laursen CB, et al. Risk factors for hospitalization due to community-acquired sepsis - a populationbased case-control study. PLoS One 2015;10:e0124838.

10 Grion CMC, Cardoso LTQ, Perazolo TF, et al. Lipoproteins and CETP levels as risk factors for severe sepsis in hospitalized patients. Eur $J$ Clin Invest 2010;40:330-8.

11 Havranek EP, Mujahid MS, Barr DA, Mujahid Mahasin S, Barr Donald $A$, et al. Social determinants of risk and outcomes for cardiovascular disease. Circulation 2015;132:873-98.

12 Ferrario MM, Veronesi G, Kee F, et al. Determinants of social inequalities in stroke incidence across Europe: a collaborative analysis of 126635 individuals from 48 cohort studies. J Epidemiol Community Health 2017;71:jech-2017-209728--2016.

13 Coughlin SS. Social determinants of breast cancer risk, stage, and survival. Breast Cancer Res Treat 2019;177:537-48. 